\title{
THE MICRO-ORGANISM OF INDIAN SUPERFICIAL PUNCTATE KERATITIS
}

BY

\author{
LT.-Col. H. HERBERT, I.M.S.(retd.)
}

THIS note is somewhat overdue on the question of the pathogenic agent responsible for the widespread and persistent epidemics of punctate keratitis seen in India. Presumably the same specific organism may account also for isolated cases of similar disease seen not only in India, but also in other parts of the world.

The question was raised afresh as part of the admirably comprehensive and far-reaching clinical, pathological and bacteriological investigation of the disease, by Lt-Col. R. E. Wright and co-workers in Madras, published in the Brit. Jl. of Ophthal., Vol. XIV (1930), pp. 257-282. It will be remembered that experimental evidence was there given pointing to a filter-passing virus as the infective agent of the disease, linking it with herpes simplex of the cornea in its varied forms.

Wright and I are agreed that the disease investigated in Madras is essentially the same as that of my old Bombay reports, published in The Ophthalmic Review, Vol. XX (1901), pp. 339-345, and in The Ophthalmoscope, Vol. II (1904), pp. 8-9. There is no doubt also that Kirkpatrick's account in the Brit. Jl. of Ophthal., Vol. IV (1920), pp. 16-20, dealt with some phases of the same epidemic disease.

How is it that in all the 30 years since my first report no widely known ophthalmologist has endorsed my finding of a causative encapsuled bacillus, except the late Prof. Axenfeld, to whom I sent a number of slides, stained and unstained? Apparently no one in India has done any work at all on the origin and spread of the disease since my efforts, except those above mentioned, and I feel sure that the failure of the Madras workers to find the organism that I had described is mainly due to my failure to emphasize the extremely limited distribution of the organism in, or on, the corneal epithelium. I hope that it is still not too late to induce someone in India, with the requisite facilities and opportunities, to utilize and to extend the following observations.

The bacillus appears to be confined entirely to the more active points of the disease, as defined by fluorescein staining, and its very feeble staining qualities add greatly to the difficulty in finding it. The search was found to be altogether too tedious unless confined solely to cases of the disease presenting the more closely packed, or the larger staining spots.

The work was certainly facilitated by the use of a stronger solution of fluorescein than the usual one. A saturated solution was 
made in a rather stronger solution of sodium bicarbonate than was laid down (I forget the precise strength). Two or three instillations were made into the patient's eye, and their effect seemed to be enhanced by an intervening cocaine instillation, restricting lacrimal secretion.

The selective colouring of active points thus secured was distinctly more effectual, and more useful, than with weaker fluorescein solutions. The aid thus given was particularly noticeable in some of the " disciform " phases of the disease, presenting a rounded central area of diffuse haziness, with defined raised margin.

The closely packed bacilli appeared to lie exclusively at the surface of the epithelium, in a single layer or little more. Their total number was evidently quite small, compared with disease of uniform distribution. Accordingly, in taking epithelial scrapings for examination light touches were made at the stained spots with the point of an old broad needle, well blunted by repeated passage through the flame, and the scanty material was well broken up on a moistened slide.

One could only hope to find one or more groups of organisms lying on, or in epithelial cells, among a large number of bacteriafree cells. Doing the whole of the work myself of collecting, fixing and staining the material, I was able in early days to estimate only "two or three" complete failures to find the bacilli in the examination of at least two or three dozen eyes. If there had been unlimited time at my disposal, probably there would have been no failures.

The most certain stain for determining the presence of the bacilli was by Gram's method, with incomplete differentiation, leaving a good deal of the tissue much overstained, and without the use of a counter-stain. But the characteristic features of the organisms were not shown thus. The capsules were not sharply outlined at all; apparently they stained more or less with the central portions. Unfortunately, I failed to seize the opportunity for closer study of the bacillus by the use of varied counterstaining after the Gram stain. The most characteristic results were obtained by the use for 10 or 15 minutes; or more, of a pale diluted borax methylene blue solution, followed by a similarly diluted eosin solution.

The colourless capsules were then sharply defined from their pink surroundings, and easily measurable. The much less defined blue centres varied in size with the depth of the blue staining. A strong impression was given that the sharp-edged capsule represented an integral part of the organism, differing essentially, for instance, from the very variable capsule of the Morax-Axenfeld bacillus. 
Dr. Surveyor, of Bombay, gave the average size of the bacillus, defined by the outer edge of the capsule, as $1.6 \mu \times 3 \cdot 2 \mu$. Axenfeld gave practically the same measurement of the bacillus, viz. $15 \mu \times 3 \mu$. It is unthinkable that this universally recognized authority and acute observer should have accepted the aetiological significance of the organism as "very probable" without good reason. In his illustrated description in "The Bacteriology of the Eye,"' MacNab's translation, 1908, pp. 325-6, he remarked that "these quite peculiar bacteria . . . were only stained with great difficulty . . . in the ordinary stains were merely hinted at, and were only demonstrable on account of their well-defined capsules."

To sum up, I personally have no doubt whatever that the bacillus is the living infective agent of the disease. I believe that it is always present in the active phases of the keratitis, and, so far as I know, it seems to be a unique organism, with unmistakable features. Its distribution-its presence in relatively large numbers at the stained spots, and there only-cannot be readily explained away.

What is the present position? How can the above findings and statement be reconciled with the Madras experimental production of the disease in human eyes in five out of eleven attempts, by rubbing in filtrates of epithelial emulsion obtained from diseased corneae, usually after scratching the epithelium of the eyes upon which the implanting was to be done? The proportion of successful implantations in these attempts with filtrates was the more noteworthy, in that it was not lower than was attained in a smaller series of similar attempts (three successful in a total of seven attempts on unaffected human eyes) made with unfiltered epithelial suspension from diseased eyes. The presumption of an active living filterable virus seems very strong from these results, and yet it must be wrong if the natural deduction from my work is to be accepted.

What is to be the outcome of this conflicting evidence? The solution of the problem calls for further investigation in both directions. In the first place renewed search for the encapsuled bacillus should be a simple matter if the disease is still epidemic in India, or when it returns. If material is available I am quite sure that any really careful and determined bacteriologist can find the bacillus as regularly as I did, using the precautions and technique given above. And something more might be learnt about the organism.

Some facts bearing upon the problem may be briefly reviewed.

There has been remarkably little evidence of transmission of the disease from patient to patient during epidemics. Do not the strongly-defined capsules suggest an exceptional resistance of the organism to drying, in its existence away from the human eye? 
A natural existence apart from the eye is perhaps suggested also by its failure to penetrate deeply into the corneal epithelium. Can the widely-spread disease be largely air-borne? An obvious argument against this is the comparative rarity of binocular affection. But this difficulty is to some extent overborne by the recognition that some slight traumatism, or some slight conjunctival irritation or conjunctivitis may be largely instrumental in implanting the infection. Wright says "Trauma is noted in the history of a large number of cases and it is quite likely that it favours an attack." In my earlier report the fact was noted that in the only case of well-marked simultaneous double punctate keratitis that had been seen, subacute trachoma was present also.

It is necessary to recall here my one experience in experimental production of the disease, briefly mentioned in The Ophthalmic Review.

At one time cultures of bacillus pyocyaneus were apparently grown in Bombay from some eyes affected with the keratitis. So far as we could do so, we excluded the possibility that these growths-very unusual from corneal surfaces-may have represented "outside" contaminations. One of the cultures was implanted on the everted upper lid of a hospital attendant. This produced a couple of small pustules followed by a curious greyish persistent palpebral conjunctival patch.

Six weeks after the implantation the cornea of this eye was found to have a mild attack of punctate keratitis. It was only a very poor development, recognizable at first by two typical staining points only. But it was undoubtedly the real thing, no less developed than in many eyes seen regularly in the out-patient department. It may have existed for some time unnoticed.

Whatever may have been the exact relationship between the pyocyaneus implantation and the subsequent slight keratitis, there is little doubt that the connection was in some way, and in some degree, an aetiological one, since this was the only eye of anyone connected with the hospital, that was ever attacked by punctate keratitis in my experience.

Apart from the remote possibility of a symbiotic relationship between the $b$. pyocyaneus and the encapsuled bacillus of punctate keratitis, the incidents related may be regarded perhaps as merely another example of trauma or conjunctivitis "favouring an attack" of the keratitis during a widely prevalent epidemic. That is, the attack probably presented merely a slight variation from many of those seen regularly among the out-patients at that time.

But this limited success, such as it was, of our single experiment prompts the inevitable inference. In how many of the more strikingly successful Madras experiments may not the same explanation have sufficed fully and truly? Can it possibly have 
been the true explanation in all? The rapid and early developments in the successful Madras cases may be held to correspond merely with the direct corneal traumatism inflicted in these experiments-scratching and massage.

Controls appear to be needed consisting of exactly similar traumatism at an epidemic period, without the use of any epithelial emulsion from diseased eyes, but possibly with the application of emulsions, filtered and unfiltered, taken from healthy eyes.

\title{
OCULAR PLUMBISM IN CHILDREN
}

\author{
BY \\ J. LOCKHART GiBSON \\ HONORARY CONSULTING OPHTHALMOLOGIST, \\ BRISBANE HOSPITAL FOR SICK CHILDREN
}

(Summary.-The cases dealt with in this paper have no pyrexia. The mild ones are brought by their mothers because they have developed a recent internal squint. The squint is due to paralysis of one or of both external recti. Papilloedema up to six dioptres is found, due to increase of intra-cranial tension. The severe cases are more evidently cases of lead encephalopathy. Explanation is given of why soluble lead is available, and why and how it is ingested. Lead is found in the urine. No albumin is found in the urine.)

Although I have written on this subject in Australia, I have only published one paper in England, and my knowledge has increased and become more accurate since then.

The peculiar nature of the cases described has not as a rule been thoroughly recognized. They appear to have no parallel among cases of industrial lead poisoning, and nearly all writers on lead poisoning where the eyes are implicated, have had experience of industrial lead poisoning only, and of that in adults. My reason for frequent references to the cases I have seen in Queensland has been the desire of myself and of my colleagues, especially of those at the Hospital for Sick Children, to get a danger removed from our young children between the ages of two years and eight years; and also to make isolated cases in other places and other countries less likely to be undiagnosed as due to lead-to bring lead at least into the differential diagnosis of such cases in young children.

It has been difficult for those practising in other parts of Australia to recognize the special conditions under which our children live, and the special facilities they have for ingesting lead. It has proved quite impossible for many of those who have investigated industrial lead poisoning to grasp the fact that whereas industrial lead poisoning occurs owing to the inhalation of lead dust, our children are poisoned owing to the ingestion of 